\title{
Fever, rash, and eosinophilia - early signs of angioimmunoblastic T-cell lymphoma
}

\author{
Bartłomiej Wawrzycki ${ }^{1, A-D, F \oplus}$, Andrzej Prystupa ${ }^{2, C-F \oplus}$, Justyna Szumiło ${ }^{3, C, F \oplus}$, Lech Panasiuk ${ }^{4, E-F \oplus}$, \\ Dorota Krasowska ${ }^{1, C, F} \odot$
}

\begin{abstract}
${ }^{1}$ Chair and Department of Dermatology, Venerology and Paediatric Dermatology, Faculty of Medicine, Medical University, Lublin, Poland

${ }^{2}$ Chair and Department of Internal Diseases, Faculty of Medicine, Medical University, Lublin, Poland

${ }^{3}$ Chair and Department of Clinical Pathomorphology, Faculty of Medicine, Medical University, Lublin, Poland

${ }^{4}$ Department of Internal Diseases and Hypertension, Institute of Rural Health, Lublin, Poland

A - Research concept and design, B - Collection and/or assembly of data, C - Data analysis and interpretation,

$D$ - Writing the article, $E$ - Critical revision of the article, F- Final approval of the article
\end{abstract}

\begin{abstract}
Wawrzycki B, Prystupa A, Szumiło J, Panasiuk L, Krasowska D. Fever, rash, and eosinophilia - early signs of angioimmunoblastic T-cell lymphoma. Ann Agric Environ Med. 2021; 28(3): 525-530. doi: 10.26444/aaem/140594

Abstract

Angioimmunoblastic T-cell lymphoma (AITL) is an uncommon lymphoma of elderly adults with a poor prognosis. AITL patients show systemic symptoms, lymphadenopathy, and not infrequently, skin rash with various dysimmune phenomena rashes. The case is presented of a 68-year-old male with skin rash, lymphadenopathy and hypereosinophilia who, after investigations, was diagnosed with AITL. Despite the treatment used, the patient's condition gradually deteriorated and died due to heart and kidney failure. The diagnosis of AITL is often established only after several weeks or months because of transient physical findings, non-specific symptoms, and a broad range of serologic or radiologic abnormalities. Some patients with AITL experience non-specific dermatitis and eosinophilia. The presented case should raise awareness of the presentations of AITL which is important for physicians to reach an accurate diagnosis.
\end{abstract}

\section{Key words}

lymphoma, eosinophilia, rash, fever, immunoblastic lymphadenopathy, hypereosinophilic syndrome, non-Hodgkin, exanthema

\section{INTRODUCTION}

Angioimmunoblastic T-cell lymphoma (AITL) is a peripheral T-cell lymphoma (PTCL) which, according to the 2016 revised WHO classification, belongs to nodal PTCL with a Follicular Helper (TFH) phenotype [1]. AITL accounts for only 1-2\% of all cases of non-Hodgkin lymphomas [2]. After excluding primary cutaneous lymphomas and lymphomas with leukemic blood involvement, the overall incidence of AITL reaches $18.5 \%$ [3]. AITL is an aggressive neoplasm with a low 5 -year survival of approximately $30-33 \%$ in most reported patient series $[3,4,5]$. AITL is a disease of elderly adults in their sixth or seventh decade of life with median age ranging from $65-69$ years $[3,5]$. At presentation, most patients $(80-$ 90\%) have advanced-stage III - IV disease by the Ann Arbor classification $[3,5]$. Some patients with AITL experience nonspecific dermatitis and eosinophilia and might be referred to an allergist/internist. The case is presented to raise awareness of the presentations of AITL which is important for physicians to reach an accurate diagnosis.

\section{CASE REPORT}

The case is presented of a 68-year-old male with exacerbated chronic obstructive pulmonary disease (COPD), heart failure,

\footnotetext{
Address for correspondence: Bartłomiej Wawrzycki, Chair and Department of Dermatology, Venerology and Paediatric Dermatology, Faculty of Medicine, Medical University, Lublin, Poland

E-mail: bartekwawrzycki@o2.pl

Received: 26.04.2021; accepted: 26.07.2021; first published: 19.08.2021
}

and type 2 diabetes mellitus, skin rash, lymphadenopathy and hypereosinophilia. According to the patient, the symptoms started three weeks before admission to our department. He recorded a rash, low-grade fever, abdominal pain, and diarrhea. Initially, the patient was hospitalized in a district hospital with a suspicion of bacterial sepsis, where he received systemic antibiotics. He was subsequently admitted to the Department of Internal Diseases. The medical history revealed hypertrophic cardiomyopathy, cardiac pacemaker implantation, percutaneous coronary intervention (PCI) on the left anterior descending (LAD) artery coronary because of coronary heart disease, and an episode of cerebral stroke. On admission, the patient presented a widespread rash consisting of erythematous and dusky papules, infiltrated plaques over the trunk, and proximities (Fig. 1a). There was also an admixture of purpuric lesions over the lateral aspects of the torso (Fig.1b). Other abnormalities were enlargement of supraclavicular lymph nodes, lower limb oedema, rattles at the lungs' base, and abdominal distension. During hospitalization, the patient complained about abdominal pain and diarrhea.

Peripheral blood testing demonstrated leucocytosis up to $23.60 \mathrm{~K} / \mathrm{uL}$ (reference range 4.0-10.0) with hypereosinophilia $3.960 \mathrm{~K} / \mathrm{uL}$ (reference range $0.0-0.5$ $\mathrm{K} / \mathrm{uL}$ ), anaemia (haemoglobin $11.1 \mathrm{~g} / \mathrm{dl}, \mathrm{RBC} 3.98 \mathrm{M} / \mathrm{uL}$, MCV $82.5 \mathrm{fl}(80.0-94.0 \mathrm{fl})$ ) and reticulocytosis - 3.13\% (0.50-2.00\%). The levels of beta 2 macroglobulin and lactate dehydrogenase (LDH) were elevated: $10.52 \mathrm{mg} / 1$ (reference range $0.7-3.0)$ and $635 \mathrm{IU} / 1$ (10-480 IU/l), respectively, as well as imflammatory markers: sedimentation rate (SR) - up to $85 \mathrm{~mm} / \mathrm{h}$ (reference range $2-35 \mathrm{~mm} / \mathrm{h}$ ) and C-reactive 


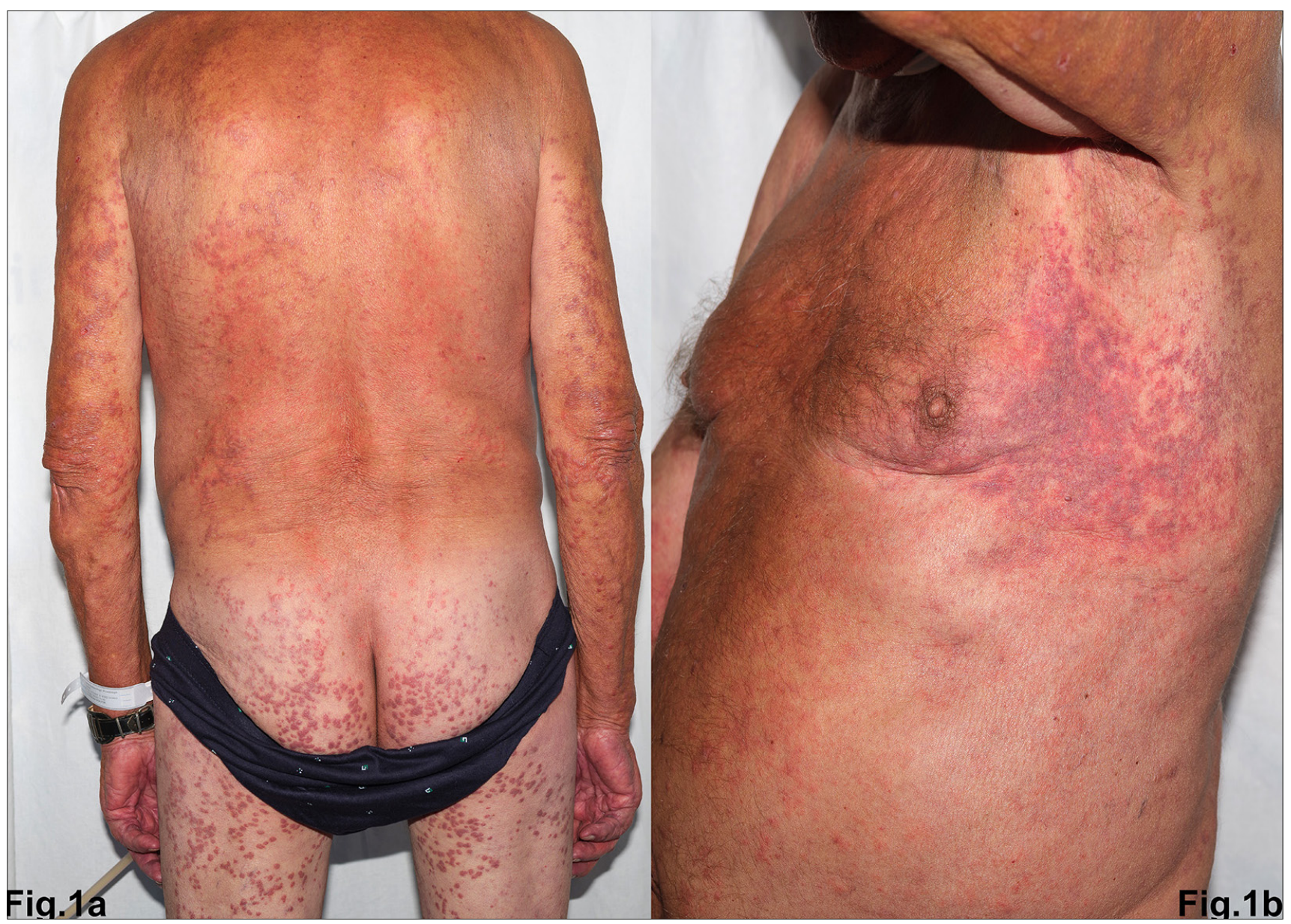

Figure 1. Widespread rash consisting of erythematous and dusky papules infiltrated plaques over the trunk and proximities (Fig. 1a). There was also an admixture of purpuric lesions over the lateral aspects of the torso (Fig. 1b)

protein (CRP) - $86 \mathrm{mg} / \mathrm{l}(<5 \mathrm{mg} / \mathrm{l})$. Serum electrophoresis with immunofixation showed: alpha 1 globulin $7.7 \%$ (2.9$4.9 \%)$, alpha 2 globulin $12.4 \%$ (7.1-11.8\%), gamma globulin $17.7 \%(11.1-18.8 \%)$, beta 1 globulin $6.1 \%(4.7-7.2)$, beta 2 globulin $21.8 \%(3.2-6.5 \%)$. On immunofixation, there was weak monoclonal IgG lambda protein. Total IgE level concentration was $8569 \mathrm{IU} / \mathrm{ml}(0-100 \mathrm{IU} / \mathrm{ml})$. The levels of individual immunoglobulins are presented in Table 1. The creatinine level was $2.6 \mathrm{mg} / \mathrm{dl}$.

Multiple blood and urine cultures were negative. C. Difficile was not detected in the qualitative faecal test. The level of procalcitonin was $0.56 \mathrm{ng} / \mathrm{ml}$.

The patient underwent chest and abdominal computed tomography $(\mathrm{CT})$, which demonstrated numerous enlarged mediastinal lymph nodes (the largest one up to $25 \times 32 \mathrm{~mm}$, axillary $(18 \times 26 \mathrm{~mm})$, and cervical lymph nodes (up to $11 \mathrm{~mm}$ ), periaortic, iliac, and inguinal lymph nodes. There were also bands of parenchymal densities in the right and left lungs.

Cervical lymph node and skin biopsy were performed for histopathological testing. The lymph node histopathology showed angioimmunoblastic T-cell lymphoma (AITL), pattern 3 (Fig. 2 a-d). The immunohistochemical stainings of skin samples were also suggestive of cutaneous T-cell lymphoma (Fig. 3 a-d).

The myelogram showed an increase in the percentage of eosinophilic granulocytes and decreased percentage of red blood cells.
Table 1. Levels of individual immunoglobulins in the presented patient

\begin{tabular}{lccc}
\hline Immunoglobulin & Result & Reference range & Units \\
\hline $\operatorname{lgE}$ & & $(0-100)$ & $\mathrm{IU} / \mathrm{ml}$ \\
\hline $\lg \mathrm{A}$ & 9.06 & $(0.845-4.99)$ & $\mathrm{g} / \mathrm{l}$ \\
\hline $\lg \mathrm{g}$ & 11.4 & $(6.10-16.16)$ & $\mathrm{g} / \mathrm{l}$ \\
\hline $\lg \mathrm{M}$ & 3.35 & $(0.35-2.42)$ & $\mathrm{g} / \mathrm{l}$ \\
\hline
\end{tabular}

Electrocardiography disclosed atrial fibrillation, while echocardiography disclosed left ventricular hypertrophy. The global systolic function of the left ventricle was normal. The level of NT-proBNP increased from $5155 \mathrm{pg} / \mathrm{ml}$ on admission to $15162 \mathrm{pg} / \mathrm{ml}(0-352 \mathrm{pg} / \mathrm{ml})$ due to progressing heart failure.

Despite the treatment used, the patient's condition gradually deteriorated and he died due to heart and kidney failure.

\section{DISCUSSION}

AITL in most patients presents as a subacute or acute systemic illness often associated with generalized lymphadenopathy, skin rash, and/or hepatosplenomegaly [6]. Generalized lymphadenopathy and B symptoms are present in 76-99 and $55-77 \%$ of patients, respectively [2]. Lymph node enlargement visualized by computer tomography is often modest $(1-3 \mathrm{~cm})[2,7] .60 \%$ of patients have bone marrow 
involvement [8]. Hepatosplenomegaly is found in one-third of affected individuals, and two or more extranodal sites are involved in at least one-fifth of cases [9]. In the presented case, $\mathrm{CT}$ results were in line with those reported in the literature the largest mediastinal lymph nodes reached up to $30 \mathrm{~mm}$ in diameter. There was no hepatosplenomegaly or bone marrow involvement; the skin was the only biopsy-proven extranodal site of involvement.

Regarding laboratory results at diagnosis, between 33-65\% of patients are anaemic [8]. Other common findings include polyclonal, hypergammaglobulinemia, especially of the IgG type - (30-65\%) [8], elevated inflammatory markers 67\% [6], elevated LDH $(60-71 \%)[3,6]$, elevated B2-microglobulin $(22-82 \%)[3,6]$ and hypereosinophilia (32-34\%) [2]. The presented patient had anaemia, elevated inflammatory markers, increased levels of LDH, B2-microglobulin, and hypereosinophilia. He had normal gamma globulins with concurrent monoclonal dysproteinemia, which is found in only $10 \%$ of patients [2].

Another peculiar laboratory finding was a high level of total serum immunoglobulin E (tsIgE). Scarce data suggest that total IgE levels could be elevated in almost half of AITL patients $[10,11]$. Recent genetic findings demonstrated a T Follicular Helper (TFH) signature of AITL [12]. TH cells localized in B-cell follicles in the secondary lymphoid organs regulate antibody isotype switching, affinity maturation, and B-cell memory generation. Nowadays, IL-4+ TFH cells are believed to be indispensable for IgE production [13]. Thus, aberrant TFH activity could be responsible for the dysimmune phenomena encountered in AITL, including IgE hypergammaglobulinemia [14].

Unfortunately, AITL symptoms may suggest autoimmune, infectious, or allergic diseases, which leads to delay of diagnosis (median time 3.6 months) [6]. Many of the patients reported in the literature also have an antecedent history of antibiotic intake [6]. The presented case exemplifies the possible diagnostic challenges posed by AITL patients. The disease onset was heralded by relatively non-specific findings - a widespread cutaneous rash, low-grade fever, moderate peripheral lymphadenopathy, and abdominal complaints with diarrhea.

On admission to the Department for Internal Diseases, the skin lesions were the most prominent sign. Cutaneous manifestations are the most common extranodal expressions in AITL patients occurring in up to $50 \%$ of cases [15] and not infrequently are the presenting complaint (up to $70 \%$ of patients) [16]. The skin involvement by AITL is polymorphic and often non-specific, commonly consisting of maculopapular eruption that may mimic drug reaction or viral exanthema $[6,16,17]$. In general, there are three main categories of skin findings in AITL macular, papular, plaque-like- nodular lesions [15], or mixed features of those above [18]. In a recent retrospective analysis, $48.8 \%$ of AITL patients had skin involvement, most frequently in the form of a non-specific rash (57.1\%), followed by papular (23.8\%), erythrodermic (16.7\%), nodular (9.5\%) and petechial/ purpuric (7.1\%) efflorescents [17]. The presented patient had a combination of papules, indurated plaques, and haemorrhagic lesions (Fig. $1 \mathrm{a}$-b). Reports on the effect of skin involvement in the survival of patients with AITL vary. Some noted that skin rash was associated with shorter survival $[19,20]$.

Diagnosis of AITL is typically based on lymph node biopsy. In the presented patient, the picture of lymph node biopsy was consistent with a diagnosis of AITL (Fig. 1 a-d) (pattern III, AITL without follicles). Characteristic nodal features of AITL like capillary hyperplasia and atypical lymphocytes with clear cytoplasm are rare in cutaneous biopsies. Two skin biopsies taken from infiltrated papule and purpuric skin lesion showed dense perivascular and sparse periadnexal dermal infiltration of medium-sized lymphocytes, which corresponded with the involvement of the skin by the AITL (Fig. 3 a-d). Histopathologically, cutaneous AITL (cAITL) shows dermal, perivascular infiltrates of small and mediumsized lymphocytes with no or minimal epidermotropism. Less frequently, the neoplastic cells are also located periadnexally [18].

The differential diagnosis of a patient with a skin rash, eosinophilia, and systemic symptoms is broad. Drug reaction with eosinophilia and systemic symptoms (DRESS) and lymphocytic variant-HES are two differential considerations in such cases. DRESS is a rare, potentially life-threatening, adverse drug reaction characterized by skin rash, fever, lymphadenopathy, haematological abnormalities, and internal organ involvement $[21,22]$. Strikingly, some AITL patients may even meet the DRESS criteria. However, in contrast to DRESS, angioimmunoblastic T-cell lymphoma patients show low drug causality probability, lesser hepatic involvement, more large-sized lymphadenopathy, and more frequent splenomegaly [23]. In the presented case, neither medication history nor liver injury was consistent with DRESS syndrome. Next, the patient's skin involvement pattern did not correspond to the typical morbilliform rash in DRESS syndrome. Finally, mediastinal lymphadenopathy, which raised concern for lymphoma in the presented case, has been rarely reported in DRESS [24].

Hypereosinophilic syndrome (HES) is a multisystem disease with a broad spectrum of cutaneous, pulmonary, and less frequently, gastrointestinal, cardiac, and neurologic manifestations [25]. HES is a diagnosis of exclusion after neoplastic, infectious, autoimmune, and drug-related hypersensitivity etiologies have been ruled out [25]. It can involve nearly every organ system and develop insidiously over time, or present acutely and progress rapidly [26]. Lymphocytic-variant of HES (LV-HES) is a subtype of HES defined by the detection of the immunophenotypically aberrant, usually monoclonal T-cell population [27]. Most patients have elevated IgE [28]. Skin histology is often uncharacteristic, and morphologic changes in the lymph nodes could mimic AITL [29]. Thus, the differential diagnosis with AITL is not always easy. The discussed patient had histopathologically confirmed lymphoma, excluding HES. In contrast to LV-HES, AITL is a disease of older individuals with a more aggressive course. Cutaneous manifestations of LV-HES include pruritus, angioedema, urticaria, eczematous dermatitis, and papular lesions. Patients with LV-HES rarely suffer from palpable purpura and the indurated plaques described in the presented case [30]. In doubtful cases, staining with TFH markers on lymph node biopsy should aid the correct diagnosis [29]. Unfortunately, at that time, such immunofluorescence markers for TFH cells were unavailable in the hospital where the patient was treated.

A variety of conditions may be accompanied by eosinophilia. Overall, allergy/atopy or drug hypersensitivity and helminth infections are the first and second most prevalent reasons for eosinophilia, respectively [31, 32, 33 ], but usually result in mildly elevated AEC (less than 


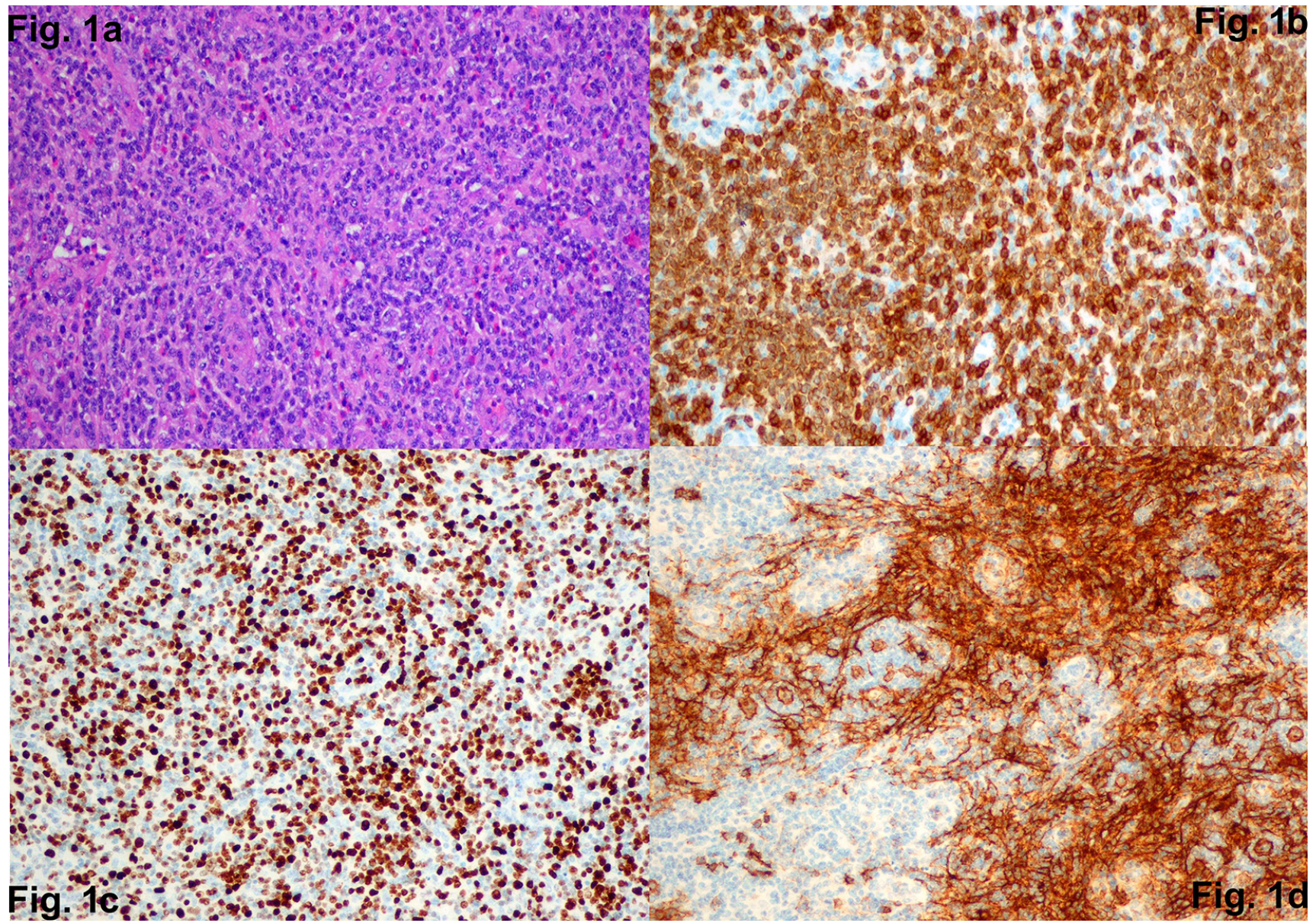

Figure 2. (a) Lymph node involvement in angioimmunoblastic T-cell lymphoma; (b) Immunoexpression of CD3 in lymphoma cells with (c) high proliferative index (Ki67); (d) Immunoexpression of CD23 proliferation of follicular dendritic cells meshwork (objective magnification a, b, c, d-×20)

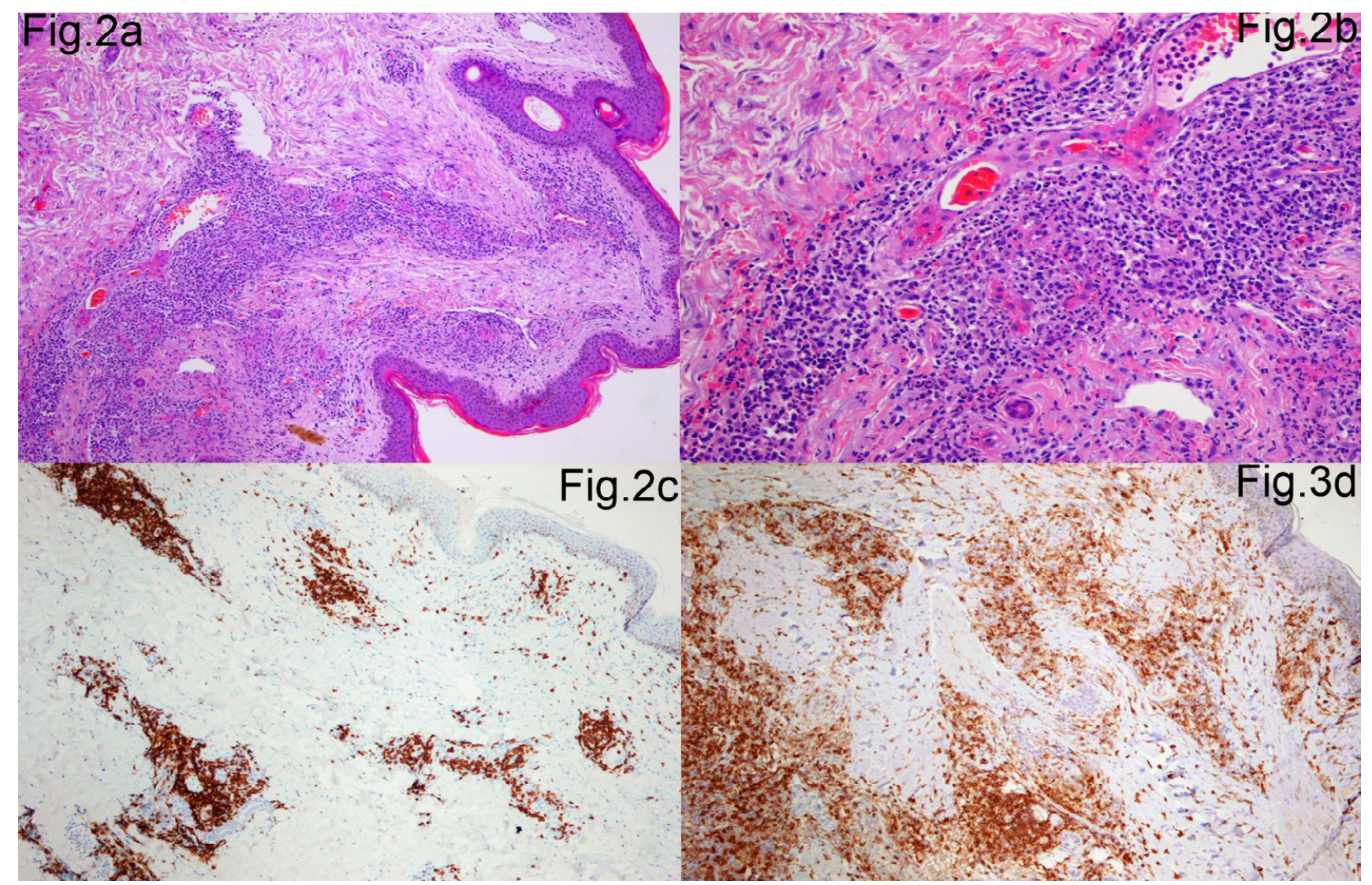

Figure 3. (a-b) Skin involvement in angioimmunoblastic T-cell lymphoma. Immunoexpression of (c) CD3 and (d) CD4 in lymphoma cells (objective magnification a, $c$, $d-\times 5, b-\times 20$ 
$1.5 \times 109 / 1)$ [34]. However, the higher the eosinophile count, the higher the incidence of hypereosinophilia-associated disease. Hypereosinophilia (HE) is defined by a marked increase in eosinophils in PB, $\geq 1.5 \times 109 / \mathrm{L}$ [35]. The presented patient had substantially elevated numbers of eosinophils, fulfilling the criterion for hypereosinophilia. Eosinophilia can accompany haematological malignancies either as a primary clonal condition or as a secondary, reactive process [36]. Non-myeloid malignancies may be associated with secondary eosinophilia driven by the abnormal production of cytokines and growth factors, usually by the tumour cells [29, 37]. Published case series revealed that the severity of initial eosinophilia makes a significant difference in the odds ratio of being diagnosed with a haematologic malignancy [36, 38]. In a study by Jin et al. [36], the overall frequency of haematologic malignancy among adults with eosinophilia was $0.2 \%$, but raised substantially to $5.1 \%$ in hypereosinophilia. In the same study, AITL was the most prevalent entity among non-Hodkin's lymphomas (NHLs) encountered in patients presenting with eosinophil counts above $1,5 \times 10$ 9/L [36]. Overall, an increased number of eosinophils is seen in onethird of the AITL cases at the time of diagnosis [2].

\section{CONCLUSIONS}

HE encompasses a broad range of differential diagnoses and may require consultations from various specialists (e.g. haematology, dermatology, pulmonology, gastroenterology, and neurology). Initial workup should determine the onset, duration, and magnitude of the eosinophilia, together with associated symptoms [39]. A detailed history of recent exposures to infections and medications is mandatory. Systemic symptoms, such as fever, night sweats, and weight loss, may suggest malignancy [39]. In any doubtful case of skin rash and lymphadenopathy, early lymph node biopsy is advisable to exclude the possibility of AITL.

\section{REFERENCES}

1. Swerdlow SH, Campo E, Pileri SA, et al. The 2016 revision of the World Health Organization classification of lymphoid neoplasms. Blood. 2016; 127(20): 2375-90. doi: 10.1182/blood-2016-01-643569

2. Lunning MA, Vose JM. Angioimmunoblastic T-cell lymphoma: the many-faced lymphoma. Blood. 2017; 129(9): 1095-1102. doi: 10.1182/ blood-2016-09-692541

3. Federico M, Rudiger T, Bellei M, et al. Clinicopathologic characteristics of angioimmunoblastic T-cell lymphoma: analysis of the international peripheral T-cell lymphoma project. J Clin Oncol. 2013; 31(2): 240-6. doi: 10.1200/JCO.2011.37.3647

4. Ermann DA, Vardell VA, Silberstein PT. Angioimmunoblastic T-Cell Lymphoma: Patient Characteristics and Survival Outcomes. Blood. 2019; 134(Supplement_1): 2194-2194. doi: 10.1182/blood-2019-132077

5. Xu B, Liu P. No survival improvement for patients with angioimmunoblastic T-cell lymphoma over the past two decades: a population-based study of 1207 cases. PLoS One. 2014; 9(3): e92585. doi: 10.1371/journal. pone.0092585

6. Lachenal F, Berger F, Ghesquieres H, et al. Angioimmunoblastic T-cell lymphoma: clinical and laboratory features at diagnosis in 77 patients. Medicine (Baltimore). 2007; 86(5): 282-292. doi: 10.1097/ MD.0b013e3181573059

7. de Leval L, Gisselbrecht C, Gaulard P. Advances in the understanding and management of angioimmunoblastic T-cell lymphoma. Br J Haematol. 2010; 148(5): 673-89. doi: 10.1111/j.1365-2141.2009.08003.x

8. de Leval L, Parrens M, Le Bras F, et al. Angioimmunoblastic T-cell lymphoma is the most common T-cell lymphoma in two distinct
French information data sets. Haematologica. 2015; 100(9): e361-4. doi: 10.3324/haematol.2015.126300

9. Broccoli A, Zinzani PL. Angioimmunoblastic T-Cell Lymphoma. Hematol Oncol Clin North Am. 2017; 31(2): 223-238. doi: 10.1016/j. hoc.2016.12.001

10. Ohsaka A, Saito K, Sakai T, et al. Clinicopathologic and therapeutic aspects of angioimmunoblastic lymphadenopathy-related lesions. Cancer. 1992; 69(5): 1259-67. doi: 10.1002/cncr.2820690531

11. Chernova NG, Soboleva NP, Mariina SA, et al. Immunoglobulinopathies in patients with angioimmunoblastic T-cell lymphoma. Ter Arkh. 2018; 90(7): 51-56. doi: 10.26442/terarkh201890751-56

12. Zhang Y, Lee D, Brimer T, Hussaini M, Sokol L. Genomics of Peripheral T-Cell Lymphoma and Its Implications for Personalized Medicine. Mini Review. Front Oncol. 2020; 10(898): 898. doi: 10.3389/fonc.2020.00898

13. Yao Y, Chen CL, Yu D, Liu Z. Roles of follicular helper and regulatory T cells in allergic diseases and allergen immunotherapy. Allergy. 2021; 76(2): 456-470. doi: 10.1111/all.14639

14. Chiba S, Sakata-Yanagimoto M. Advances in understanding of angioimmunoblastic T-cell lymphoma. Leukemia. 2020; 34(10): 25922606. doi: 10.1038/s41375-020-0990-y

15. Botros N, Cerroni L, Shawwa A, et al. Cutaneous manifestations of angioimmunoblastic T-cell lymphoma: clinical and pathological characteristics. Am J Dermatopathol. 2015;37(4): 274-83. doi: 10.1097/ DAD.0000000000000144

16. Martel P, Laroche L, Courville P, et al. Cutaneous involvement in patients with angioimmunoblastic lymphadenopathy with dysproteinemia: a clinical, immunohistological, and molecular analysis. Arch Dermatol. 2000; 136(7): 881-6. doi: 10.1001/archderm.136.7.881

17. Lee WJ, Won KH, Choi JW, et al. Cutaneous angioimmunoblastic T-cell lymphoma: Epstein-Barr virus positivity and its effects on clinicopathologic features. J Am Acad Dermatol. 2019; 81(4): 989-997. doi: 10.1016/j.jaad.2018.08.053

18. Oishi N, Sartori-Valinotti JC, Bennani NN, et al. Cutaneous lesions of angioimmunoblastic T-cell lymphoma: Clinical, pathological, and immunophenotypic features. J Cutan Pathol. 2019; 46(9): 637-644. doi: $10.1111 /$ cup. 13475

19. Archimbaud E, Coiffier B, Bryon PA, Vasselon C, Brizard CP, Viala JJ. Prognostic factors in angioimmunoblastic lymphadenopathy. Cancer. 1987; 59(2): 208-12. doi: 10.1002/1097-0142(19870115)59:2<208::aidcncr2820590205>3.0.co;2-7

20. Siegert W, Nerl C, Agthe A, et al. Angioimmunoblastic lymphadenopathy (AILD)-type T-cell lymphoma: prognostic impact of clinical observations and laboratory findings at presentation. The Kiel Lymphoma Study Group. Ann Oncol. 1995; 6(7): 659-64. doi: 10.1093/ oxfordjournals.annonc.a059281

21. Husain Z, Reddy BY, Schwartz RA. DRESS syndrome: Part I. Clinical perspectives. J Am Acad Dermatol. 2013; 68(5): 693 e1-14; quiz 706-8. doi: 10.1016/j.jaad.2013.01.033

22. Kardaun SH. Drug Reaction with Eosinophilia and Systemic Symptoms (DRESS). In: Shear NH, Dodiuk-Gad RP, eds. Advances in Diagnosis and Management of Cutaneous Adverse Drug Reactions. Springer Singapore; 2019: 87-104: chap Chapter 7.

23. Jeong J, Sim DW, Yu JE, et al. Differentiation of angioimmunoblastic T-cell lymphoma from DRESS syndrome. J Allergy Clin Immunol Pract. 2019; 7(5): 1684-1686 el. doi: 10.1016/j.jaip.2018.11.048

24. Werbel T, Castrovinci P, Contestable J. Ethosuximide-induced drug reaction with eosinophilia and systemic symptoms with mediastinal lymphadenopathy. Pediatr Dermatol. 2019; 36(4): e99-e101. doi: 10.1111/ pde.13848

25. Eng V, Kulkarni SK, Kaplan MS, Samant SA, Sheikh J. Hypereosinophilia with angioimmunoblastic T-cell lymphoma. Ann Allergy Asthma Immunol. 2020; 124(5): 513-515. doi: 10.1016/j.anai.2020.01.028

26. Hsieh FH. Hypereosinophilic syndrome. Ann Allergy Asthma Immunol. 2014; 112(6): 484-8. doi: 10.1016/j.anai.2014.03.004

27. Khoury P. Lymphocytic-Variant Hypereosinophilic Syndromes. Hematologist. 2017; 14(6). doi: 10.1182/hem.V14.6.7884

28. Ogbogu PU, Bochner BS, Butterfield JH, et al. Hypereosinophilic syndrome: a multicenter, retrospective analysis of clinical characteristics and response to therapy. J Allergy Clin Immunol. 2009; 124(6): 1319-25 e3. doi: 10.1016/j.jaci.2009.09.022

29. King RL, Tan B, Craig FE, et al. Reactive Eosinophil Proliferations in Tissue and the Lymphocytic Variant of Hypereosinophilic Syndrome. Am J Clin Pathol. 2021; 155(2): 211-238. doi: 10.1093/ajcp/aqaa227

30. Marzano AV, Genovese G. Eosinophilic Dermatoses: Recognition and Management. Am J Clin Dermatol. 2020; 21(4): 525-539. doi: 10.1007/ s40257-020-00520-4 
31. Sade K, Mysels A, Levo Y, Kivity S. Eosinophilia: A study of 100 hospitalized patients. Eur J Intern Med. 2007; 18(3): 196-201. doi: 10.1016/j.ejim.2006.09.031

32. Thompson Coon J, Pittler M, Ernst E. Herb-drug interactions: survey of leading pharmaceutical/herbal companies. Arch Intern Med. 2003; 163(11): 1371. doi: 10.1001/archinte.163.11.1371-a

33. Peju M, Deroux A, Pelloux H, Bouillet L, Epaulard O. Hypereosinophilia: Biological investigations and etiologies in a French metropolitan university hospital, and proposed approach for diagnostic evaluation. PLoS One. 2018; 13(9): e0204468. doi: 10.1371/journal.pone.0204468

34. Klion AD. How I treat hypereosinophilic syndromes. Blood. 2015; 126(9): 1069-77. doi: 10.1182/blood-2014-11-551614

35. Wang SA. The Diagnostic Work-Up of Hypereosinophilia. Pathobiology. 2019; 86(1): 39-52. doi: 10.1159/000489341
36. Jin JJ, Butterfield JH, Weiler CR. Hematologic Malignancies Identified in Patients with Hypereosinophilia and Hypereosinophilic Syndromes. J Allergy Clin Immunol Pract. 2015; 3(6): 920-5. doi: 10.1016/j. jaip.2015.06.009

37. Shomali W, Gotlib J. World Health Organization-defined eosinophilic disorders: 2019 update on diagnosis, risk stratification, and management. Am J Hematol. 2019; 94(10): 1149-1167. doi: 10.1002/ajh.25617

38. Andersen CL, Siersma VD, Hasselbalch HC, et al. Eosinophilia in routine blood samples and the subsequent risk of hematological malignancies and death. Am J Hematol. 2013; 88(10): 843-7. doi: 10.1002/ajh.23515

39. Khoury P, Bochner BS. Consultation for Elevated Blood Eosinophils: Clinical Presentations, High Value Diagnostic Tests, and Treatment Options. J Allergy Clin Immunol Pract. 2018; 6(5): 1446-1453. doi: 10.1016/j.jaip.2018.04.030 